\title{
RECURRENT NEURAL NETWORK FOR CLASSIFYING OF HPC APPLICATIONS
}

\author{
Trong-Ton Pham \\ Mathieu Pister \\ Philippe Couvée \\ Bull - Atos Technologies \\ HPC Software R\&D \\ 1 rue de Provence, 38432 \\ Echirolles, France \\ \{trong-ton.pham, mathieu.pister, philippe.couvee\}@atos.net
}

\begin{abstract}
This paper presents a novel approach using deep neural networks in HPC software application domain. The approach uses Recurrent Neural Network (RNN) and more specifically Long Short-Term Memory (LSTM) models to classify jobs running on HPC clusters into different application families. This is a mandatory step in order to optimize their run time environment for our future work. Our approach was inspired by the recent advancement in Deep Learning in the AI area. We implemented our models on a HPC cluster and took advantage of the GPU to accelerate the training process. The evaluation of our models on a database of synthetic jobs (with more than 500 runs) gave good results for the classification of short duration jobs (less than 1 hour). In comparison with the conventional approaches such as Support Vector Machine and Hidden Markov Model, the stateful LSTM implementation improves significantly the classification accuracy for long duration jobs (more than 2 hours).
\end{abstract}

Keywords: Classification, RNN, LSTM, Deep Learning, Exascale

\section{INTRODUCTION}

Next-generation of exascale supercomputers will require more and more automation (Abrahm 2015) to overcome growing complexity in their administration and usage. All levels of supercomputer architecture will be affected by automation, such as power consumption, network, data storage, etc.

Nowadays, automated solutions are already in use to predict breakdown events (Gainaru 2013) in data centers. However, next generation solutions will be able to, not just predict events, but also automatically reconfigure the supercomputer to maintain operating performance in an optimal state. Such reconfigurations, that will affect all levels of the supercomputer, require an on-line monitoring of metrics and a dynamic adjustment of its operating state.

Such software products are developed in our lab in the area of $\mathrm{I} / \mathrm{O}$ accesses on supercomputers. Depending on the application behavior, I/O access can be optimized using accelerators such as data prefetchers or burst buffers. Our goal is to automate the setting up of the optimal I/O environment for an application, based on previous runs analysis. To do so, we develop 3 products that work together and make a continuously improving cycle as described in Figure 1:

- I/O Instrumentation: an application I/O profiler for HPC applications.

- I/O Pattern Analyzer: a data analytics tool for classification of multiple jobs and accelerators automation. 

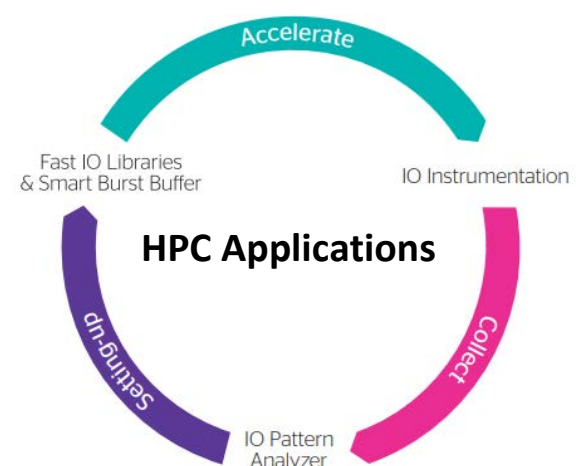

Figure 1: Continuous improvement of I/O efficiency in HPC applications with three products: Instrumentation, Pattern Analyzer and Accelerators.

- Fast I/O Libraries and Smart Burst Buffer: I/O accelerators targeting various I/O patterns such as pseudo random read and burst writes.

Classifying jobs into different application families is a crucial task for the I/O Pattern Analyzer product. First, it helps to classify automatically runs with similar behaviors into a same group, so we can apply the right accelerator and the optimized parameters for that job family. Second, it helps to better identify outlier runs that deviated from the job family (for example, run of job that takes much longer to finish caused by network or disk access problems). These outliers can help to do root cause analysis of the failures in the data center. This work aims to solve this important point on the data management side.

Deep learning (Goodfellow 2016), as part of Machine Learning revolution, has transformed different areas of science and engineering. These techniques have been applied in several domains such as image classification (Yoshua 2012), face recognition (Parkhi 2015) and speech translation (Graves 2013), etc. Current processing power of HPC computers and dedicated hardware (such as GPU or FPGA) continues to growth in the recent years (https://www.nvidia.com/en-us/data-center/machine-learningbenchmarks/). It makes it possible and more affordable to train the deep neural network in terms of performance and cost efficiency.

Recurrent Neural Network (RNN) (Schmidhuber 1993) and Long Short-Term Memory (LSTM) (Hochreiter 1997) are specific variants of Neural Network. This model has been adopted widely for sequence learning and machine translation. One major advantage of RNN is the idea that it can memorize the previous information for the current task. In theory, RNN are capable of handling such "long-term dependency". However, as the sequence grows, RNN becomes unable to learn correctly the connection of the information. This "long-term dependency" problem is also known as gradient vanishing problem (Hochreiter 2001). LSTM reduces this problem by introducing a new type of neural cell state which allows controlling the information pass through the cell.

To our knowledge, this is the first approach in the HPC domain that uses RNN for job modeling and classification. Compared to other conventional approaches such as Support Vector Machine (SVM) (Cortes 1995) and Hidden Markov Model (HMM) (Baum 1966), we obtained better results in term of accuracy. We present our main contribution in this work as follows:

- Introduce the recurrent neural network and LSTM models into the classification of HPC applications based on the collected I/O metrics.

- Improve the capacity of training the model for long sequence by introducing the new batch representation of the stateful LSTM model.

- Demonstrate the performance of this approach over the classical classification methods such as SVM and HMM.

In the Section 2, we provide some backgrounds on the RNN and LSTM network model. We explain how we applied those models for classification of short and long jobs. Then, we discuss in detail about our implementation of the stateless and stateful LSTM networks in Section 3. In the Section 4, a job database was created based on a simulation of synthetic HPC application to validate the results of our implementation. We give some insights on the result of the proposed methods and compare it to the 
other approaches for classification tasks. Finally, we conclude the paper in Section 5 and give some perspectives about the future work.

\section{DEEP NEURAL NETWORK MODEL}

\subsection{Recurrent Neural Network (RNN)}

The Recurrent Neural Network was developed to model time series data (Che 2016). The structure of RNN is similar to the Multiple Layer Perceptron (MLP) but with a simpler representation for a sequence as input data. Each element of a sequence is passed to the RNN one by one to train the model. RNNs can use their internal state (memory) to process sequences of inputs. Through the connections among the hidden units between time steps, the model can retain information about the past elements. This enables the discovery of the temporal correlation between the sequences or time series data. This is a crucial property for learning sequence data where the occurrence of one element might depend on the presence of other elements in the sequence.

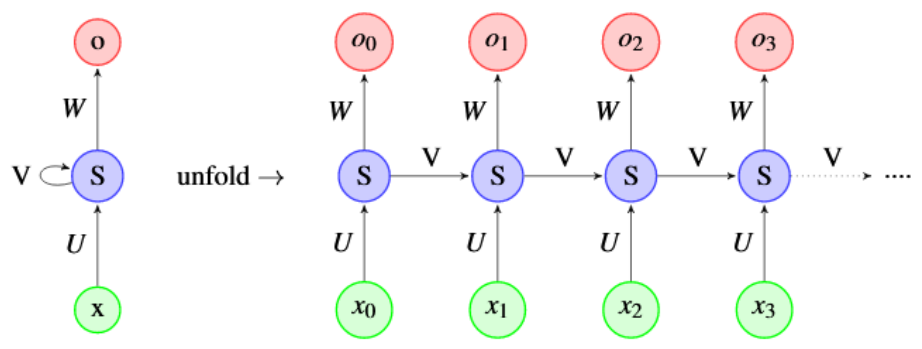

Figure 2: A recurrent neural network unfolded for a sequence $\mathrm{x}$.

A basic recurrent neural network is initialized with a state vector $\mathbf{s}_{\mathbf{0}}$, usually consisting of only zeros. A sequence $\mathbf{x}$ of input vectors $\mathbf{x}_{\mathbf{t}}$ is indexed by time step $\mathbf{t}$ (see Figure 2). The length of the input vector can be variable depending on the length of a sequence. The RNN then runs through a sequence of state vectors $\mathbf{s}$, determined by the following formula:

$$
\mathbf{s}_{\mathbf{t}}=\mathbf{W} \boldsymbol{\sigma}\left(\mathbf{s}_{\mathbf{t}-1}\right)+\mathbf{U} \mathbf{x}_{\mathbf{t}}+\mathbf{b},
$$

where the trainable parameters of the model are the recurrent weight matrix $\mathbf{W}$, the input weight matrix $\mathbf{U}$ and the biases $\mathbf{b}$. The model parameters $\boldsymbol{\theta}=(\mathbf{W}, \mathbf{U}, \mathbf{b})$ are learned by minimizing the cost function with a gradient-based optimization method. A cost function measures the performance of the network on some given task after having seen $\mathbf{t}$ input vectors and transitioning to state $\mathbf{s}_{\mathbf{t}}$.

The mapping from an input sequence to a state sequence is typically referred to as a recurrent layer in neural network terminology. RNNs can have several of such layer stacked on top of each other.

\subsection{Long Short-Term Memory (LSTM)}

In principle, the RNN is a simple and powerful model. In practice, it is hard to train properly with gradient descent method. Among the many reasons, there is a major problem that have been referred as the vanishing gradient problem. This problem refers to the fact that gradients can decrease exponentially as the length of the input sequence grows. Very small gradients cause the network to learn very slowly or even to stop completely. Therefore, LSTM has been introduced by Hochreiter et al. in 1997 to unravel this problem.

LSTM introduces a new neural cell state to the basic RNN structure. The cell state is like a conveyor belt in the neural network. It runs straight down the entire chain, with only some minor linear interactions. It is very easy for information to just flow along it unchanged. The LSTM does have the ability to remove or add information to the cell state, regulated by structures called gates.

Gates are a way to optionally let information go through. They are composed out of a sigmoid neural network layer and a pointwise multiplication operation. The sigmoid layer outputs numbers between zero and one, describing how much of each component should be let through. An LSTM has three of these gates, to protect and control the cell state. A detailed explanation of the LSTM network is beyond 
the scope of this paper, interested reader may refer to the tutorial in Colah's blog (http://colah.github.io/posts/2015-08-Understanding-LSTMs/).

\section{JOBS CLASSIFICATION MODEL}

\subsection{Feature engineering}

RNNs allow to use different length sequences. Due to the training by batches, all sequences in a batch must have the same length. Therefore, we apply the padding function to add extra timesteps to the sequence so that all sequence has the same length. The values added to the vectors are negative because they do not represent real values.

We can consider the job run as a sequence of 5-second time steps. For each time step, 10 metrics have been extracted to build the feature set from the $\mathrm{I} / \mathrm{O}$ metrics collected in the database (for example: $\mathrm{I} / \mathrm{O}$ volume for read and write operations, number of I/O access patterns). These features have been selected as they represent well the read/write patterns of one job and they are distinctive enough to differentiate 2 runs of a same job. Due to the environment (node executing the code, network, file system), runs of the same job are different. The sequences are then normalized for each metric per run using min-max normalization. For each time steps, the metric values are normalized between 0 and 1 using the following formula:

$$
z_{i}=\frac{x_{i}-\min (x)}{\max (x)-\min (x)} .
$$

The normalization step is crucial as we can have a job with a very big $\mathrm{I} / \mathrm{O}$ volume and a job with moderate $\mathrm{I} / \mathrm{O}$ volume. The important information of the $\mathrm{I} / \mathrm{O}$ patterns persists during the execution with the min-max normalization.

\subsection{LSTM sequential model}

The example model used in our work is shown in Figure 3. It can be decomposed into two parts:

- The first part is composed of the 3 LSTM layers. It transforms the variable length sequences into a fixed length vector.

- The second part is a dense layer. This layer is composed of as many neurons as the number of classes.

Dropouts has been used inside each LSTMs layer to reduce the over-fitting of the network during the training. The outputs of LSTM model use softmax activation function to provide the probability for each class.

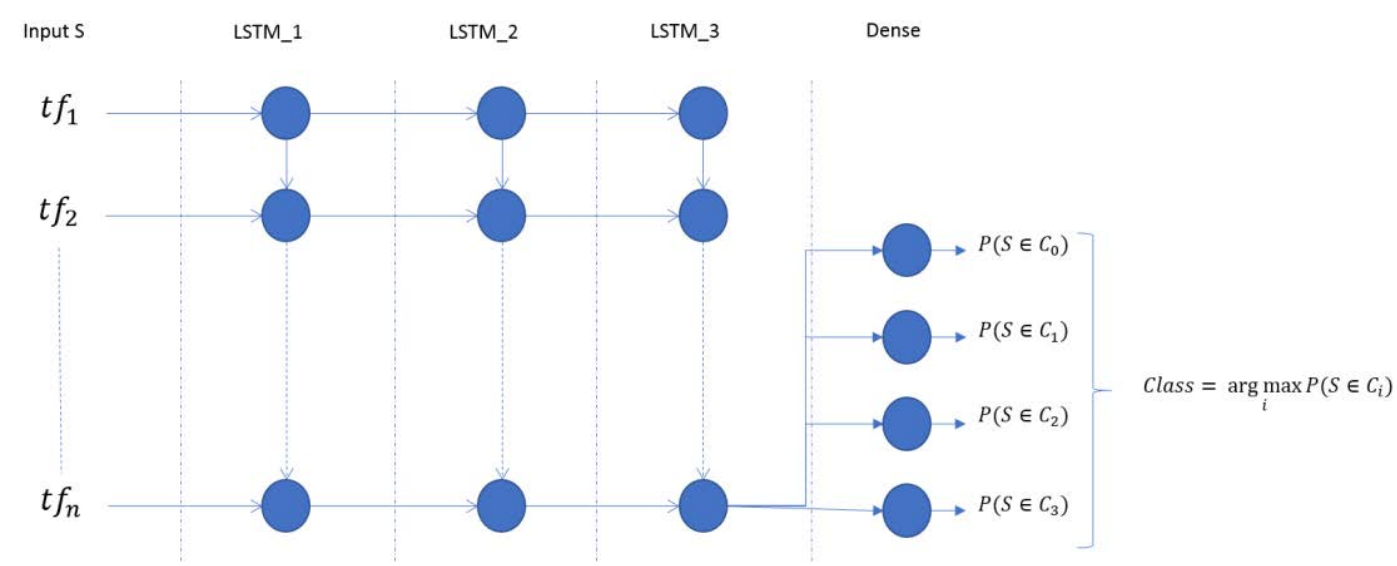

Figure 3: LSTM sequential model composed of 3 layers and a dense layer for classification aggregation. In this example, we have 4 classes corresponding with 4 neurons in the dense layer. 


\subsection{Model training}

\subsubsection{Set splitting}

The model is trained and tested separately on 3 different sets (defined in part 4). In total 520 sequences are available. Each data set is split randomly into a training set (70\%) and a test set (30\%). A validation set is taken from the training set. It represents $10 \%$ of the training set. It is used during the training to compute quality metrics of the training (i.e. loss value or accuracy). A checkpoint is used to save the weights of the model at the epoch that had the best loss value on the validation set. At the end of the training, the last checkpoint is considered as the best model for testing the performance on test set.

\subsubsection{Hardware setup}

All the experiments were run on a cluster of 3 compute nodes consist of CPU Intel Xeon (R) @ $2.40 \mathrm{GHz}$ (16 cores on 2 sockets) and 64Gb of memory. Also, we have one compute node containing 2 GPUs Nvidia grid K22 Kepler (3,072 CUDA cores) and 8 Gb GDDR5 (4Gb per GPU).

The training of neural networks is complex and the default implementations on CPU does not benefit the power of the GPUs on our clusters. Moreover, Nvidia proposed an optimized implementation of LSTM based on the cuDNN library (https://developer.nvidia.com/cudnn). Its implementation is available in the Tensorflow and Keras libraries. We compare the training time per epoch for these two implementations on both CPU and GPU in the Table 1.

Table 1: Comparing training time per epoch with different LSTM implementations on CPU and GPU.

\begin{tabular}{|l|c|}
\hline LSTM implementation & Training time per epoch \\
\hline CPU with Keras & 284 seconds \\
\hline GPU with Keras & 25 seconds \\
\hline GPU with cuDNN & 2 seconds \\
\hline
\end{tabular}

As we can see from this table, the LSTM model using cuDNN implementation gives the fastest training time on GPU over the Keras implementation on GPU and CPU. It is a big improvement compared to the standard implementation with CPU (more than 140 times faster). We finally choose the cuDNN implementation of LSTM in our experimentations.

\subsection{Implementation}

Our implementation has been built around Keras library (https://keras.io/) and TensorFlow framework (https://www.tensorflow.org/). TensorFlow is an open source software library developed by Google for machine learning and deep learning tasks. Its flexible architecture allows easy deployment of computation across a variety of platforms (CPU, GPU). Keras is a high-level neural networks API, written in Python and capable of running on top of TensorFlow. Keras also runs seamlessly on both CPU and GPU. It allows easy and fast prototyping of deep neural networks. This helps developer focus on the experimentation of the model and tuning the hyper-parameters.

Keras supports both stateless and stateful model for training LSTM networks. Figure 4 shows the difference between these two implementations. In the stateless model, the cell states are reset at each sequence. With the stateful model, all the states are propagated to the next batch. 


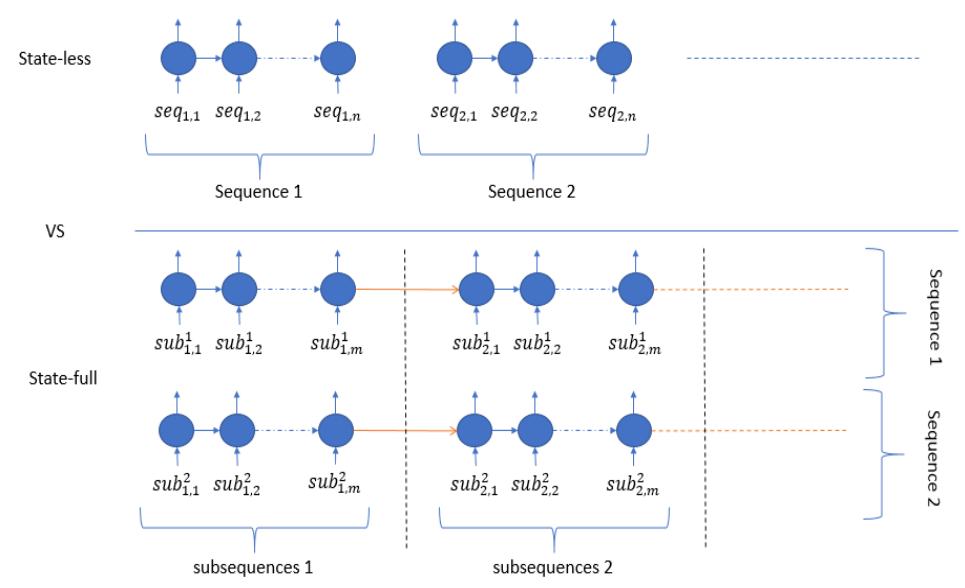

Figure 4: The difference between two implementations of stateless and stateful LSTM models are on the transferring of the learned states from one sequence (or sub-sequence) to another.

While stateless model supports training the LSTM with a batch of different sizes, the classic stateful model supports only training with a fixed batch size (either one or all the sequences). This makes the training of the stateful model in Keras extremely slow and unstable. To be able to train our stateful LSTM model with variable batch sizes, we need to reimplement the batch creation for stateful model. We will go into details of our implementation of the stateless and stateful model in the following sections.

\subsubsection{Stateless model}

In stateless model, we trained the model using mini-batches size of 32 sequences. During the training, each sequence is processed independently from each other. The state of one sequence is not transferred to the next sequence. Keras library support this implementation by default so we do not need to do extra processing on the sequences.

\subsubsection{Stateful model}

The implementation of stateful LSTM is more complex than the stateless one. Sequences need to be split into sub-sequences with the same length (called window size). In the stateful model, the computed states of previous sub-sequence are used as initial state of the next sub-sequence.

Figure 5 illustrates the creation of batches from a set of sequences. Sequences are grouped into a same group. From one batch group, the model creates a list of states to pass. This list is used as initial states for the next batch and so on. In this configuration, the order of the batches and of sub-sequences inside batches is very important. The black arrows represent the states passing from one batch of sub-sequences to another batch of sub-sequences.

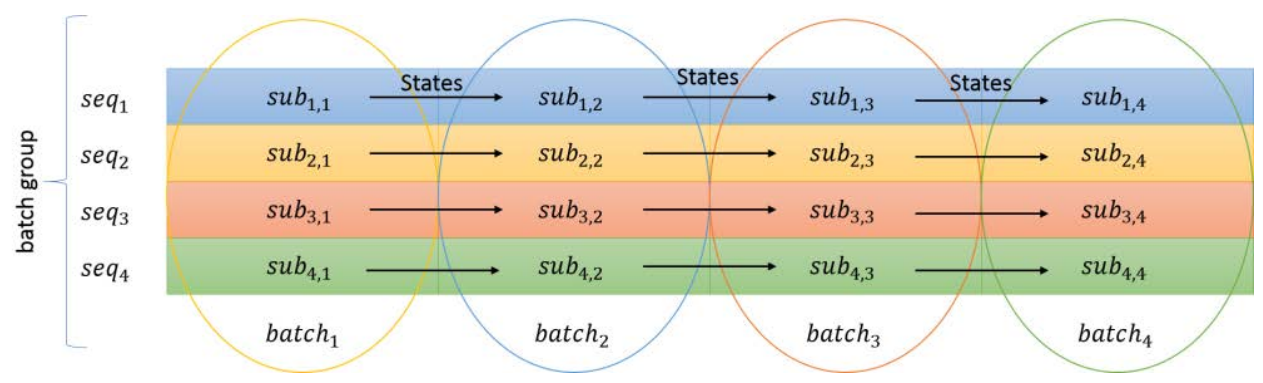

Figure 5: Creation of batch group from the sub-sequences for training stateful LSTMs.

Each sequence is then associated to a class using job name as label. When sequences are split into subsequences, each sub-sequence is then associated to the sequence's class. With this implementation, we make it possible to train LSTM network with a variable batch size in the stateful model with Keras. 


\subsection{Prediction \& accuracy}

For the stateless model, the predicted class $C_{j}$ of a sequence $S_{i}$ (or a job) is the one that maximizes the predicted probability.

For the stateful model, it can take the most predicted class for all sub-sequences $s u b_{\{i, \mathrm{k}\}}$. Or another way is to choose the class that maximizes the average probability $P\left(s u b_{\{i, k\}} \in C_{j}\right)$ over all sub-sequences.

$$
\begin{gathered}
S_{i}=\left(\operatorname{sub}_{\{i, 1\}}, \operatorname{sub}_{\{i, 2\}}, \ldots, s u b_{\{i, n\}}\right) \text { and } \\
\operatorname{Pred}\left(S_{i}\right)=\operatorname{argmax}_{j} \frac{\sum_{k=1}^{n} P\left(\operatorname{sub} b_{\{i, k\}} \in C_{j}\right)}{n} .
\end{gathered}
$$

Finally, we measured the accuracy rate using the percentage of the correct prediction over the test set.

\section{EXPERIMENTS}

\subsection{Simulation of job database}

The simulation application is a tool developed by our data management team to be able to create applications that generate I/O following different patterns (i.e. sequential, random or stride). Those $\mathrm{I} / \mathrm{O}$ patterns are parameterized following different criteria such as: I/O size, number of read or write operations. For example, it allows to create an application that simulates a check point by writing sequential block of data. It can add some $\mathrm{I} / \mathrm{O}$ ground noise (i.e. small $\mathrm{I} / \mathrm{O}$ variations) to make different variances of $\mathrm{I} / \mathrm{O}$ patterns.

With this simulation application, we can produce applications that have I/O behaviors closed to the real HPC applications. We instrumented these jobs and collected the data running in our HPC cluster. Those jobs were run in parallel in order to stress the cluster's network, the file system caches and then introduced some noise in the records. All this creates a real situation to have differences between runs of the same applications. For instance, we noticed some differences on the execution time of runs of the same job (around $1 / 4$ of the mean execution time).

Figure 6 shows an example workflow to create a simulation HPC application. The job synthetizer allows to define sub-application blocks combining with other Linux commands (sleep, $d d$, etc.). An application is defined as a set of sub-applications. The job synthesizer creates the new application following its job script.

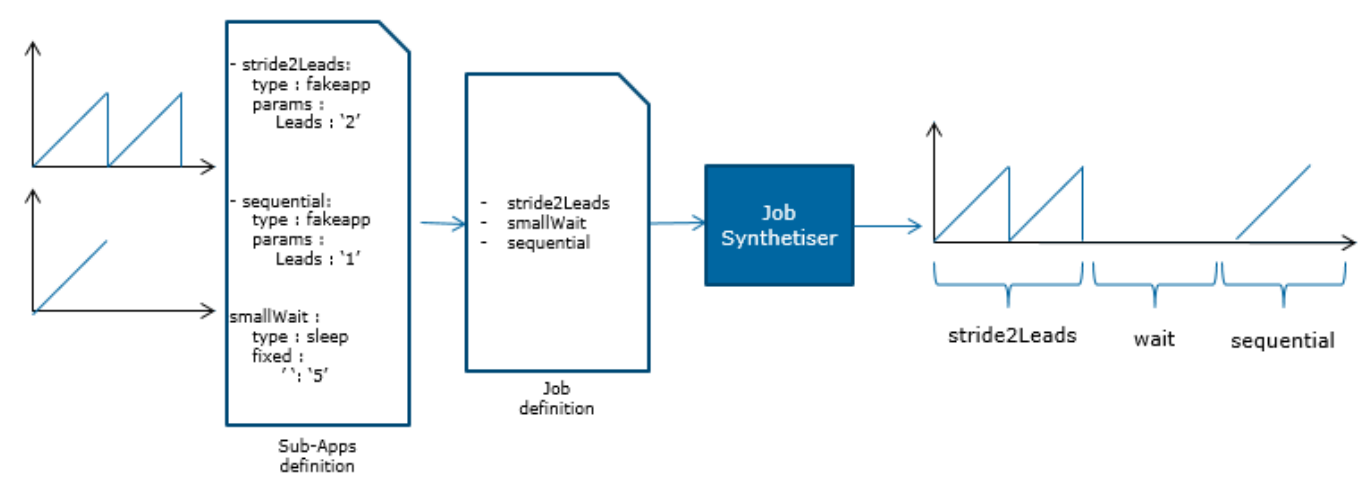

Figure 6: Job synthetizer simulates I/O behaviors of HPC application by mixing different I/O.

The idea of having different sets of jobs is to test the ability of the neural network to well classify on different jobs and on similar jobs. Also, we would like to test the impact of the job duration (i.e. short job and long job) on the classification. Each collection is composed of several job applications. For each application, we collected the metrics for 40 different runs. Those runs were launched on 3 compute nodes working in parallel on the same file system. This increases the difference between runs of the same job and makes the classification task harder. 
The first job set (Figure 7) is composed of four different jobs. They have different durations, number of $\mathrm{I} / \mathrm{O}$, volumes of $\mathrm{I} / \mathrm{O}$, number of processes, etc. They are composed of the same sub-applications, so they execute the same patterns of $\mathrm{I} / \mathrm{O}$ with the same $\mathrm{I} / \mathrm{O}$ size.

We added another job set (Figure 8) composed of three jobs quite similar in terms of I/O activities. The objective is to test the ability of the model to differentiate the jobs with a very subtle variations. These two sets have a short duration from 5 to 15 minutes per runs.

The third job set (Figure 9) was designed to test the model's limits with longer job duration. It is composed of three jobs with duration from $2 \mathrm{~h}$ to $2 \mathrm{~h} 30$. Each job is composed of two parts. The first 30-minute part has a small difference for each job. The second part is very similar across the jobs. This set should test the robustness and the capacity of memory at the beginning of job execution for the model.

In total, the data set is composed of 10 different job applications. All 520 instrumented job runs are stored in a job database. An example of run for each application is given in terms of $\mathrm{I} / \mathrm{O}$ volume read and write in each table. We use this data set to test the performance of the neural network, as well as comparing with other state of the art classifiers in the next section.

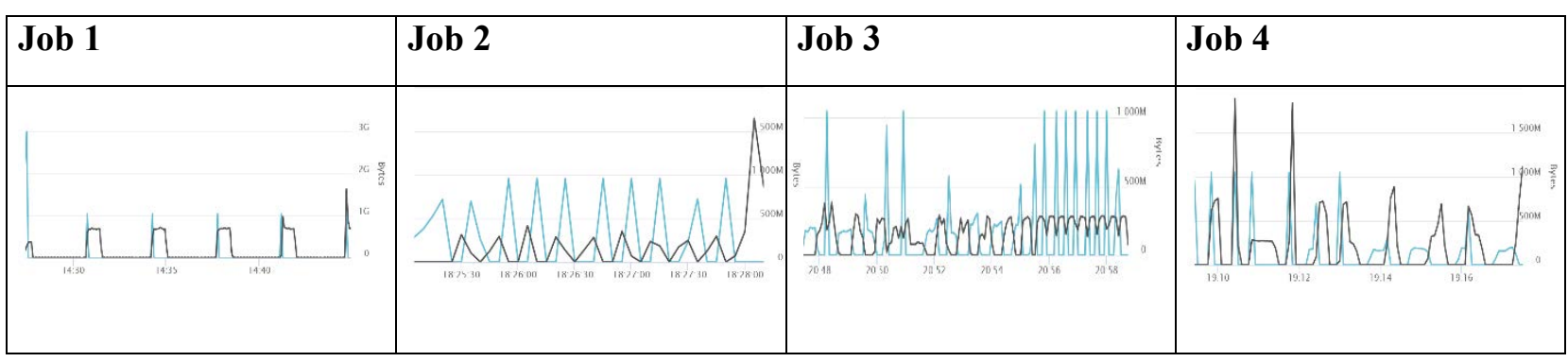

Figure 7: Example of the first dataset with 4 short jobs (5-15 minutes). Jobs are represented by the total $\mathrm{I} / \mathrm{O}$ volumes (read and write in bytes) monitored by the I/O Instrumentation of each timestep $5 \mathrm{~s}$ in the vertical axis.

\begin{tabular}{|l|l|l|l|}
\hline Job 1 & Job 2 & Job 3 \\
\hline & & \\
\hline
\end{tabular}

Figure 8: Example of the second dataset with 3 short similar jobs of 5-15 minutes.

\begin{tabular}{|c|c|c|}
\hline Job1 & Job2 & Job3 \\
\hline$\underset{1330}{\operatorname{mand}} \underset{1100}{\text { Non }}$ & $\underset{1200}{\operatorname{and}}$ & 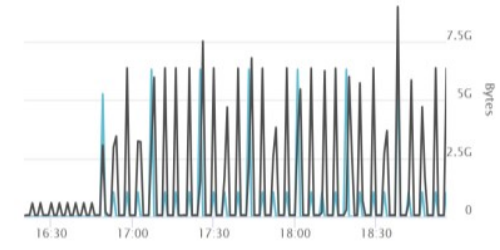 \\
\hline
\end{tabular}

Figure 9: Example of the third dataset with 3 long jobs $(2 \mathrm{~h}-2 \mathrm{~h} 30)$. 


\subsection{Results on the short job dataset}

The LSTM model is trained using the stateless model on each dataset. For the first sets, this model gave us an accuracy of $100 \%$. It is able to classify well on very different jobs with different behaviors and different duration. However, the limits of the model were reached with the third set of jobs. The first half hour is specific to each job but the remaining hour and a half are similar to the 3 jobs. Figure 10 shows the decreasing of the prediction accuracy of the stateless LSTM model as we increase the training data frames. At 400-600 time steps (30 minutes to 1 hours), the stateless model still performs quite well with the accuracy superior to $80 \%$. After that, the prediction accuracy drops drastically from $600-1000$ time steps. Over 1000 time steps, the model gives a very poor accuracy of $30 \%$ which is about the same with a random classification.

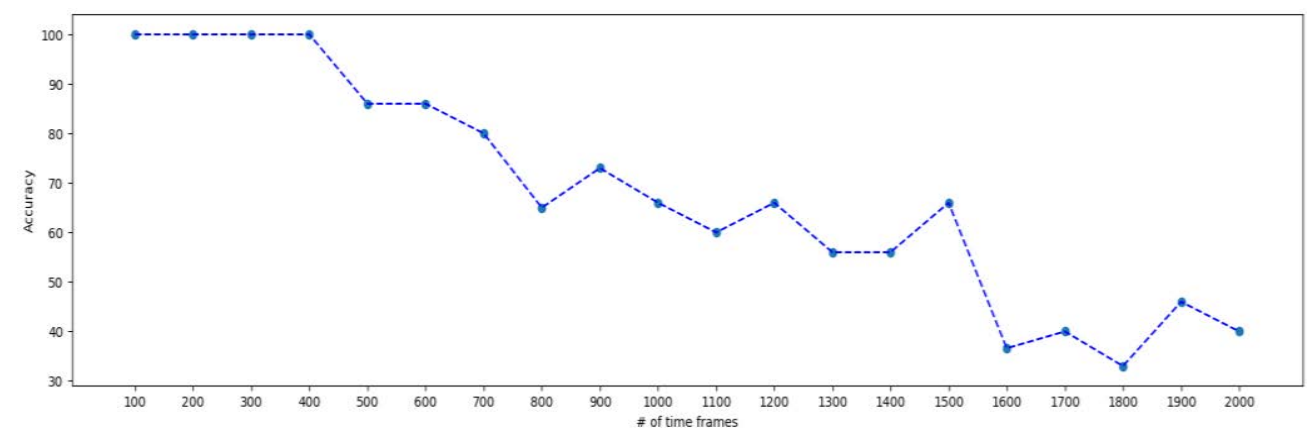

Figure 10: Accuracy of the stateless model decreases significantly over 600 time steps.

The stateless model gave a very good accuracy for this classification of short job runs. But as expected, it is inefficient for long sequences due to the gradient vanishing problem. Technically, increasing the network layers can increase the accuracy on longer sequences. However, it was not the best solution because increasing the network size will also increase the network complexity and training time. For this reason, we apply the stateful model to overcome the long-term dependencies as well as maintaining the efficiency of LSTM for the long job runs.

\subsection{Results on the long job dataset}

We run the LSTM stateful model on the long job data set with the same hyper-parameters of the network with an additional parameter on the window size. The window size is the number of time steps for each sub-sequence, i.e. the length of the sub-sequence. It is a new hyper parameter introduced with the implementation of stateful LSTM.

To observe the impact of the window size on the classification accuracy, the model was trained 15 times for each window size. To reduce changes between two trainings, every training is done using the same training set and test set. The neural network was initialized with the same starting weights as the stateless LSTM networks.

Figure 11 shows the mean of the accuracies computed for each window size. From this graph, we can see that the best window size for our data set is achieved for 400 time steps with an accuracy of $96 \%$. Window size of 200 is also a good choice with an accuracy of $92 \%$. But all the configurations of window size smaller than 200 or bigger than 450 seem to be less effective. We can also notice that for a window size of 1000 , the stateful model gives the same results as the stateless model with accuracy around $33 \%$. 


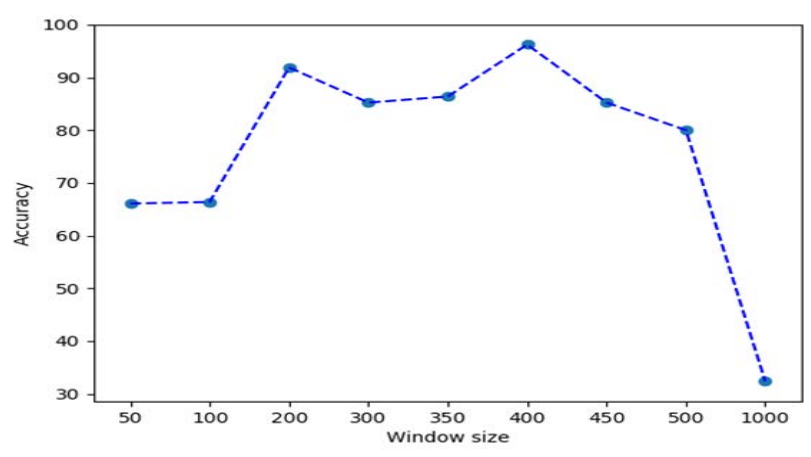

Figure 11: Effect of the window size on the mean accuracy of 15 trainings.

As we can observe from this result, the stateless LSTMs is well suited for sequence classification, but it has difficulties with long sequences due to the gradient vanishing problem. The stateful LSTM variant allows to split sequences into sub-sequences and transfer the neural states to the next sub-sequence. Therefore, it improves significantly the classification performance for longer sequences.

\subsection{Comparison with other methods}

To confirm the good performance of the LSTMs network, other state-of-the-art classification algorithms have been implemented for comparison. Two supervised learning methods have been selected in this experiment: Support Vector Machine (SVM) and Hidden Markov Model (HMM).

SVM applies the statistics to identify the support vectors that maximize the separation of the labeled data. It is one of the most widely used method for classification task. We extracted set of features from the same metrics collected as above. Those metrics are normalized and concatenated into a metrics histogram of 25 bins. Job runs are represented by a set of feature vectors and their respective classes. They are then fed into multi-class SVM to train the classifiers. We applied the Radial Basis Function (RBF) kernel for better separating non-linear data.

HMM is a generative probabilistic model. It can be considered as a generalization of mixture model in which a sequence of observable variables is generated by a sequence of internal hidden states. HMM can work directly with normalized sequences as the LSTM models but without the need for padding. We then trained one HMM classifier for each class using Viterbi algorithm. After the training, each HMM will return a score for a new sequence. This score is high if a HMM model could have generated this sequence. To decide the job of a run, we choose the HMM classifier that has the biggest score.

Table 2: Summary of the accuracy performance on different models in percentage.

\begin{tabular}{|l|c|c|c|c|}
\hline \multicolumn{1}{|c|}{ Model } & SVM & HMM & $\begin{array}{c}\text { Stateless } \\
\text { LSTM }\end{array}$ & $\begin{array}{c}\text { Stateful } \\
\text { LSTM }\end{array}$ \\
\hline Shataset job 1 & 100 & 100 & 100 & 100 \\
\hline Short job 2 & 64 & 64.7 & 100 & 100 \\
\hline Long job & 30 & 73 & 33 & 96 \\
\hline
\end{tabular}

Table 2 summarizes the results we obtained for each model on 3 different data sets. As expected, the SVM performed quite good on the short job sets but very poorly with the long job set. HMM has a quite similar behavior as SVM for the two shot jobs dataset with $100 \%$ and $64 \%$ respectively. They are both below the performance of 1LSTM model at $100 \%$ of prediction accuracy.

For the second short job set, both LSTM models outperformed the SVM and HMM. This shows the robustness and the capability of the LSTM on memorizing sequences that have a very small difference. On the long jobs, HMM gives a good prediction accuracy at $73 \%$ but only the stateful LSTM model 
reached very high prediction accuracy at $96 \%$. This result confirms the best overall prediction accuracy of the stateful LSTM network.

\section{CONCLUSION AND FUTURE WORK}

In this paper, we introduced a novel application of Recurrent Neural Network and LSTM for classification of HPC applications. During our experiment, we also noted that the application of LSTM is not as straight-forward as we might think. The default implementation of stateful LSTM is not convenient for training with different batch sizes, which is an important criterion in fine tuning the hyper-parameters of the LSTM network. We introduced a better way to train the stateful model by splitting sequences into groups of sub-sequences.

Our study demonstrated that both stateless and stateful LSTM model performed very well on a data set of short jobs (i.e. duration less than 1 hour). For the long jobs, the stateful model exhibited a significant improvement over the stateless model. We also compared the result with other baseline methods (SVM and HMM) to confirm the good performance of our stateful LSTM implementation.

Our objective in this study is to enhance the performance of the application running on HPC clusters. One promising direction is to deploy this model on a data center to benefit from the massive data collected to train our network and test the scalability of the proposed method.

Future work also includes the possibility of combining with current trend in reinforcement learning (Van Hasselt 2015) to close the loop from collecting data, classifying jobs into similar groups and automatically applying the right $\mathrm{I} / \mathrm{O}$ accelerator parameters for the job. This will create an autonomous system for monitoring and administrating HPC cluster in the next decade.

\section{REFERENCES}

Abrahm, Erika, et al. (2015). "Preparing HPC applications for exascale: Challenges and recommendations.", 18th International Conference on Network-Based Information Systems (NBiS).

Alex Krizhevsky, Ilya Sutskever, and Geoffrey E Hinton (2012). "ImageNet classification with deep convolutional neural networks". In Advances in neural information processing systems. 10971105.

Baum L. E., Petrie T. (1966). "Statistical Inference for Probabilistic Functions of Finite State Markov Chains". The Annals of Mathematical Statistics. 37 (6): 1554-1563.

Che Z., Purushotham S., Cho K., Sontag D., Liu Y. (2016). "Recurrent neural networks for multivariate time series with missing values". Nature.

Cortes Corinna, Vapnik Vladimir (1995). "Support-vector networks". Machine Learning. 20 (3): $273-$ 297

Ian Goodfellow, Yoshua Bengio and Aaron Courville (2016). "Deep Learning”. MIT Press.

Hochreiter S., Schmidhuber J. (1997). "Long Short-Term Memory". Neural Computation. 9 (8): 17351780.

Hochreiter S., Bengio Y., Frasconi P. and Schmidhuber J. (2001). "Gradient flow in recurrent nets: the difficulty of learning long-term dependencies". A Field Guide to Dynamical Recurrent Neural Networks.

Parkhi Omkar M., Andrea Vedaldi, and Andrew Zisserman (2015). "Deep face recognition". BMVC. Vol. 1. No. 3.

Gainaru Ana, et al. (2013). "Failure prediction for HPC systems and applications: Current situation and open issues." The International Journal of High Performance Computing Applications. 273-282.

Graves Alex, Mohamed Abdel-rahman, Hinton Geoffrey (2013). "Speech Recognition with Deep Recurrent Neural Networks". Acoustics, Speech and Signal Processing (ICASSP), 2013 IEEE International Conference on: 6645-6649.

Schmidhuber Jürgen (1993). "System modeling and optimization". Page 150. Habilitation thesis. 
Van Hasselt Hado, Guez Arthur, Silver David (2015). "Deep reinforcement learning with double Qlearning". AAAI Conference on Artificial Intelligence: 2094-2100.

Yoshua Bengio (2012). "Deep learning of representations for unsupervised and transfer learning". Unsupervised and Transfer Learning Challenges in Machine Learning. Page 19.

\section{AUTHOR BIOGRAPHIES}

TRONG-TON PHAM holds a PhD in Information Retrieval and Computer Science from Grenoble Institute of Polytechnics (INPG). He currently works as Data Scientist at the HPC Software R\&D in Bull-Atos Technologies. His research interest includes data modeling, machine learning and optimization research. His email address is trong-ton.pham@atos.net.

MATHIEU PISTER obtained his Master degree from ENSIMAG engineering school with an specialization in Artificial Intelligence. Currently, he works in the Cognitive Data Center group at the HPC Software R\&D in Bull-Atos Technologies on predictive maintenance. His email address is mathieu.pister@atos.net.

PHILIPPE COUVEE received an Engineer degree from University of Lille. He is currently head of Data Management group at the HPC Software R\&D in Bull-Atos Technologies. His interest lies in data storage, application performance and HPC architecture. His email address is philippe.couvee@atos.net. 